\title{
Kinematic design of a footplate drive mechanism using a 3-DOF parallel mechanism for walking rehabilitation device
}

\author{
Chu ZHANG*, Bluest LAN**, Daisuke MATSUURA*, Céline MOUGENOT*, Yusuke SUGAHARA* and \\ Yukio TAKEDA* \\ * Mechanical Systems Design Laboratory, Department of Mechanical Engineering, Tokyo Institute of Technology \\ Ookayama, Meguro-ku, Tokyo 152-8552, Japan \\ E-mail: takeda@mech.titech.ac.jp \\ ** Research Center for Advanced Science and Technology, The University of Tokyo \\ Komaba, Meguro-ku, Tokyo 153-0041, Japan
}

Received: 16 August 2017; Revised: 21 December 2017; Accepted: 24 January 2018

\begin{abstract}
Recently, facilities with the special intention to help people who lack walking ability or even sit in the wheel chair could be seen everywhere. However, in the hospitals, rehabilitation devices, which are used for people to regain their body functions right after some health problems, are very limited, and even the existing robots have not shown sufficient evidence to prove they are useful, low cost, or easy to handle to be used as a regular rehabilitation method. In this research, a 3-DOF parallel mechanism training device as a compact foot drive mechanism for the gait rehabilitation of walking-disabled patients was developed for solving these sorts of problems. The requirements and constraints were obtained through interviews with medical doctor, physical therapists and related researchers. The composition of this proposed training device is: patients can be supported by a harness system with their feet fastened onto two separated programmable footplates. Based on the preprocessed walking data captured from healthy individuals, reference motion for the footplate can be determined and used to calculate the specifications of the footplate drive mechanism. As the foot drive mechanism, a planar parallel mechanism with 3-DOF was selected. Its dimensional synthesis was carried out taking into consideration the workspace and motion transmissibility, and a prototype of the machine was designed and built in the end. Comparison between the walking data and mechanism ' $\mathrm{s}$ analysis indicated that it is possible for the machine to generate natural walking patterns, which will be further proved with a prototype in the future.
\end{abstract}

Keywords : Gait rehabilitation, Parallel mechanism, Workspace, Motion transmissibility, User centered design

\section{Introduction}

With the increasing average lifespan, facilities of special intention to help the elders who lack walking ability could be seen everywhere. The rehabilitation devices, in the hospitals, for them to regain their body functions right after some health problems are still far from enough, even the existing robots have not shown very sufficient evidence to prove they are useful, easy to handle, and good enough to be used as a regular rehabilitation method. Currently, physical therapists provide the main recovering treatment, which are very labor intensive especially for gait rehabilitation. Normally, it takes a number of forms, but repetition of the actual walking motions is the most important one. The need for high quality therapists brings heavy economic burden to hospitals especially in a country short of human resources.

When we imagine rehabilitation for patients who tore Achilles' tendon or broken lower limb bones, and had taken surgical operation, its current protocol is separated in-between early phase in which range of the damaged joint is recovered, and mid-term phase in which whole lower limb is moved so that coordinated motion among several joints without giving significant load for each joint, and later gait training phase in which patients walk on flat ground (at first, then slopes and staircases are added). In the early phase and mid-term phase, patients are lying on beds and therapists moves 
his or her damaged joint (or whole leg) so that no undesirable load is exerted. In the later gait training phase, on the other hand, patients' body weight is fully supported by their-own legs, and therapists directly move the patient's leg or standing beside the patients to avoid falling down. Because of this separation, gait training takes long time and is costful by means of time and human resource.

In order to solve this problem, we considered a compact and low cost robotic rehabilitation system that can move patients' limbs in proper trajectory while supporting his or her body. In the long term, it can contribute to start the gait training without load in the mid-term phase, and can assist therapists to treat more patients at the same time. As a result, the physical training effort of a therapist can be reduced. Building such a training device for walking rehabilitation is the main target of the research. This paper discusses a novel design of foot-driving mechanism using parallel mechanism that is capable of generating healthy gait pattern by driving one's foot while his or her body weight is supported. Based on two different methods of fixing user's foot onto a foot-plate, necessary kinematic design of the mechanism is calculated. One of the methods considers to minimize the constraint of user's foot to minimize the essential volume of the device, by utilizing human's lower limb structure to achieve natural gait pattern. The obtained calculation result is used to construct a prototype foot driving mechanism, and several user tests have been carried out to demonstrate the effectiveness of the proposed design approach.

\section{Related work and literature review}

In order to help the patients reinstate the independent walking ability, various gait training devices have been designed to replace traditional efforts. Among these devices, there are several major approaches which researchers are utilizing. The standing posture gait trainers may be the most common method and can be classified into three categories simply:

(1) Treadmill with orthosis gait trainer

(2) Footplate based gait trainer

( 3 ) Wearable exoskeleton

For each category, we would like to show one example. The Lokomat system (Hocoma AG), which consists of a gait orthosis and an advanced body weight support system (BWSS), combined with a treadmill (Hesse and Uhlenbrock, 2000). Another one is called the Gang trainer GT I, commercialized by Reha-Stim. Harness-secured patients are positioned on two-foot plates, whose movements simulate stance and swing, and ropes attached to the patient can control the vertical and lateral movements of the center of mass. Lastly, Hybrid Assistive Limb (HAL), which is a wearable robot designed for a wide range of applications, from rehabilitation to heavy work support, and built in several versions, full body version and two-leg version (Kawamoto and Sankai, 2002).

As a fact, exoskeleton without BWSS is still not safe enough and takes labor to ensure the safety of the user. In the application of this group, the patients feet suffer from pressure to support the weight. This pressure may be useful in some cases, but in other cases users need to avoid such foot pressure. While in the other two groups with BWSS, treadmill with orthosis group should control the velocity of the two sets of actuation systems synchronously very well, which need sophisticated and complex control system. In another aspect, the system is too complicated that takes too much time for people wearing. Therefore, the footplate based gait trainers may be an easier device for using. A smaller, low cost design with normal walking gait function is better and more user friendly.

\section{System design concept}

Among all the various devices that are being researched, one major approach is to make the foot attached to an end-effector generating human-like walking patterns, which was advised by medical doctors. By using this, the load of human leg is reduced partially. Combining with virtual reality technology, as shown in Fig. 1, this kind of system can give virtual environments to motivate users and make them feel less tired. Real time data can be collected through vision-based sensors, so that the actual movement of user's legs and his/hers intention behind it could be considered in the control system of the device. A programmable foot motion generator can be designed for generating several kinds of patterns and helping users to move their limbs with the correct posture. Thus, the system is aimed to meet the user 


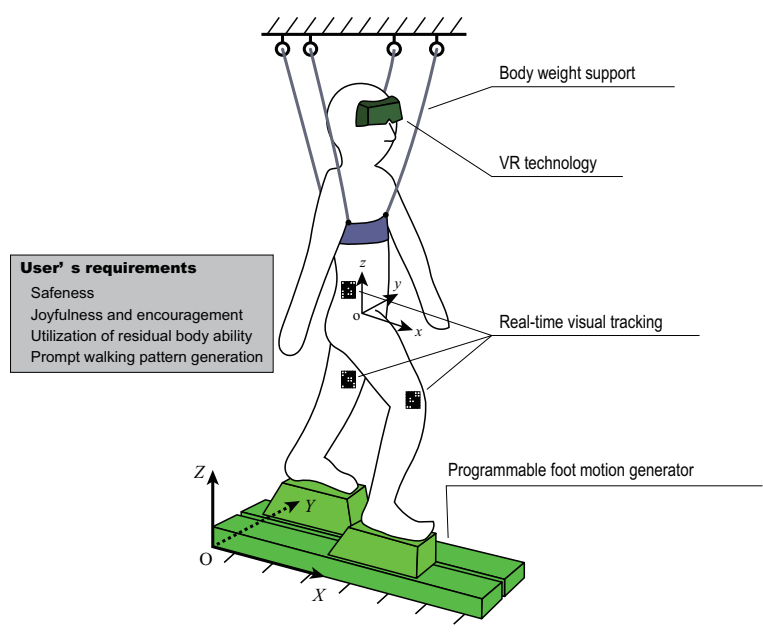

Fig. 1 The overview of the gait rehabilitation system

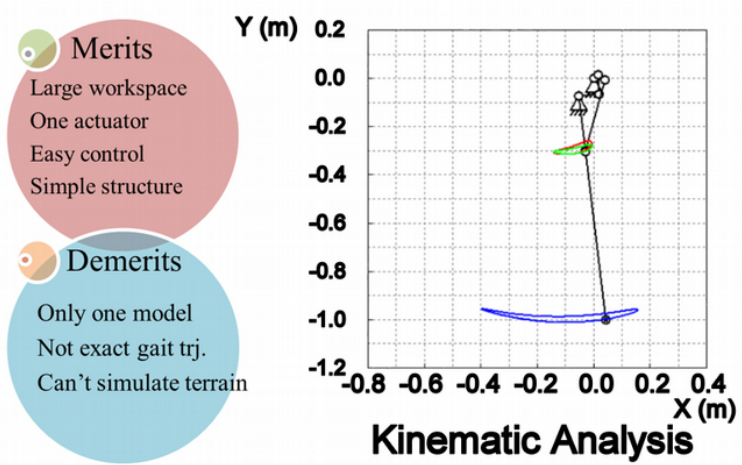

Fig. 2 Comparison of different mechanisms (Matsuura et al., 2014)

needs summarized from lots of user interviews and finally fulfill the task of gait training. To this end, we had interviews from medical doctors and physiotherapists, and had carried out measurements of the foot's motions by four independent subjects with different heights, which were reflected in the design discussed in this paper.

Regarding the design of the programmable foot motion generator, mechanical composition proposed in former researches can be classified into two groups; one is serial mechanisms, and the other is closed-loop and parallel mechanisms. Serial mechanism is used in almost all wearable- and treadmill-type devices. Advantage of this type can be summerized as large workspace and high capability of motion generation. Oppositely, risk for out-of-control and poor weight-to-payload ratio are its disadvantage. On the other hand, closed-loop or parallel mechanisms have a great deal of advantages over serial ones in load-carrying capacity, stability, precision. As an example of this category, a gait training device using a pair of 3-DOF five-bar closed-loop mechanisms can be found (Yoon et al., 2010). However, the closed-loop nature limits the motion of the platform and creates complex kinematic singularities in their workspace. As the result, rehabilitation devices using this type of mechanisms tend to become complex, and their occupation volumes are generally large against its workspace. Adding redundancy to the device might increase workspace, avoid singularity, and improve dexterity (Wang and Gosselin, 2004). However, the cost and analysis work will also be increased. Some people had tried to construct hybrid mechanisms that mount a serial mechanism on top of a closed-loop mechanism, such as Haptic Walker (Schmidt et al., 2005), but it yet occupies a large volume.

In order to construct a foot motion generator being compact, safe and enough powerful, minimally-essential mechanical condition to constraint and drive user's legs promptly should be taken into account. This can be done by considering human's body as a part of the mechanical system, and analyze whole system behavior. From this perspective, the authors' research laboratory had developed a walking assist machine that can generate leg motion to step forward only by changing the distance between a user's hip joint and ankle joint with single actuator (Matsuura et al., 2015). There was another unique feature of the machine called "foot driving mechanism", which utilizes a transformable mechanism having a lockable sliding joint to achieve non-linear and rapidly-increasing acceleration profile. The idea of transformable mechanism has been applied to another link mechanism in Fig. 2 to give different outputs by adjusting the links (Matsuura et al., 2014). The workspace is large enough and the structure is quite simple, yet the end-effector is so far that its stability cannot be maintained. Desired gait patterns cannot be created with only a simple model. The last elliptical trainer made by Adaptive Motion Trainer (AMT) (Precor Inc., accessed on 1 Janualy, 2016) is similar, the design makes walking too fierce for patients.

Based on the above discussion, the authors have come up with an idea to revise the constrained condition of user's foot on the platform, not to fully constrain the entire foot but to constrain only the forefoot instead, so that one's foot can be allowed to change its contact condition between the foot-plate while a cycle of gait, and thus human's natural walking motion can be achieved while minimizing the mechanism's essential workspace. In this way, we determined the manipulator to be a planar 3-DOF structure to generate the 3-DOF motions on the sagittal plane. Normally, three 
actuators either linear or rotational are needed on different kinematic chains. Thus, there should be two closed loops in the mechanism. Through Eq. (1), where $f_{j j}, L$ and $d$ stand for DOF of joint $j j$, number of independent loops and dimension of the motion space, respectively, the number of joints with one DOF in each kinematic chain, $J_{1}$, can be obtained.

$$
\begin{aligned}
F & =\sum_{j j=1}^{J} f_{j j}-L d \\
& =3 J_{1}-2 \times 3=3 \\
& \Rightarrow J_{1}=3
\end{aligned}
$$

Though we know that in each chain there should be three joints with one DOF, it can be prismatic joint or revolute joint. As written in (Bonev et al., 2003), we have ten planar parallel mechanisms with kinematically symmetrical structure as candidates. These are $\underline{R} R R, R \underline{R}, \underline{R R P}, \underline{R P R}, \mathrm{RPR}, \underline{\mathrm{PRR}}, \underline{\mathrm{P} R}, \underline{\mathrm{R}} \underline{\mathrm{PP}}, \underline{\mathrm{PRP}}$, and $\underline{\mathrm{PPR}}$, where $\mathrm{P}$ and $\mathrm{R}$ stand for revolute and prismatic joints respectively, and underlined symbol represents active joint.

Since the workspace of the manipulator should be enough for generating the foot trajectory, as well as avoiding singularities. Another important requirement is the occupied space of the device, which could be estimated with the trajectory size. From the Jacobian matrices, singularities of the manipulator could be studied and find the singularity free workspace for a certain orientation. However, as the orientation of the foot changes, we have to check the workspace for all the desired orientations. This is the first requirement for the mechanism design of the walking assist device. Once it is done, the leg lengths and total size of the device could be estimated and evaluated, which is the constraint for the design to help with eliminating the bad ones. The cycle goes on again and again until the best mechanism is found.

In order to increase the walking direction workspace, a nice way is making three prismatic joints align to be parallel. In this way, mechanism should start with prismatic joint; that is, PRR and PRP are the two choices. On another aspect to make the structure compact, revolute joint takes less space than prismatic joint, PRR is more compact. Thus, the final mechanism was chosen as a 3-PRR parallel structure, which has been widely used as a parallel manipulator. A redundant actuated parallel manipulator has been developed by using 4 legs (Harada and Nagase, 2009). In conceptual research, the actuation redundancy was not used since the transmission angles were kept very well and singularity can be avoided. Finally, our CAD model could be obtained and is shown in Fig. 3. Three sliders driven with ball screws were used to realize the function of prismatic joints, which were aligned parallel. Each motor would be assembled at the end of the screw in order to actuate the attached slider. Between the sliders and the end-effector, three links were connected through revolute joints. The footplate was supported by three links, two of which were symmetric and form an isosceles triangle, and the joints connected them to the footplate with the same rotation axis.

\section{Kinematic design}

In order to determine the machine specifications, motion capture system was used to get reference data. The experimental environment is shown in Fig. 4, 18 reflective markers were attached to different parts. After several tests, 4 typical data sets shown in Table 1 were chosen for advanced study.

The chosen 4 subjects' height and leg length can ensure that the machine can generate a wide range of gait patterns according to the kinematic analysis, no matter how the patients' gait trajectories change with gender, age, or even walking

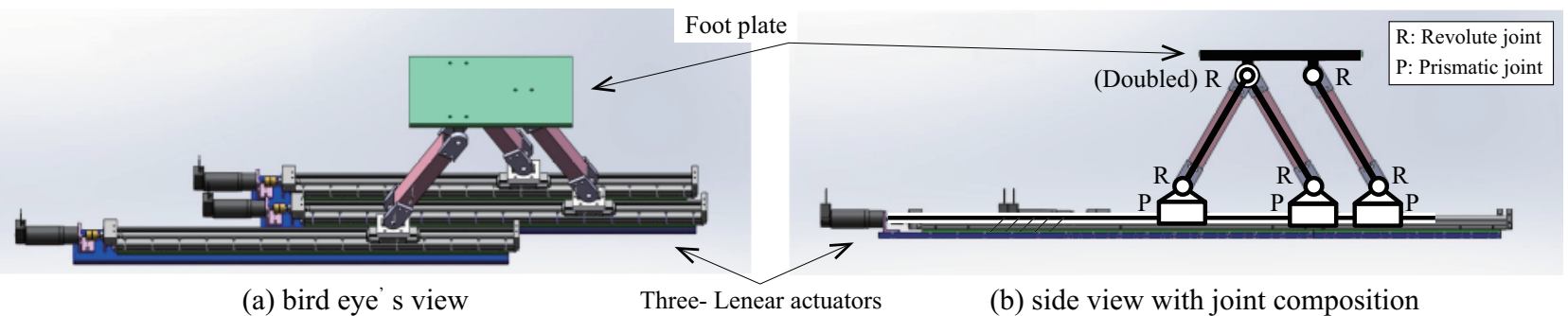

Fig. 3 CAD model of the footplate mechanism 
habits. The heel and toe trajectories during natural walking by the 4 subjects are shown in Fig. 5 .

Since how the foot is placed on the footplate will affect the calculation result, two different methods were considered with kinematic analysis. The first method, Method 1, is to attach hind foot onto the footplate (Fig. 6a), the other one, Method 2, is to keep the forefoot fastened (Fig. 6b), but the heel can be moved passively according to the posture of the leg.

Considering the first case, to calculate the workspace of the mechanism, it is necessary to know the links' length and the distance between two joints under the footplate. The coordinate system starts from the left limit that Slider 1 could reach. The right hand side is the walking direction, which is also pointing to the positive X. Upward is defined as the positive $\mathrm{Z}$ direction.

Figure 7 shows the kinematic diagram of the planar 3-PRR mechanism. This mechanism is composed of three connecting chains of PRR structure, the base link and the output link as the foot-plate. By co-axializing two of the three revolute joints on the output link by a double revolute joint, its kinematics is simplified. The inverse kinematics to determine the input displacement of sliders 1 to 3 for a given pose of the output link can be carried out as follows. When the position of $\mathrm{P}_{1}$ is given, input displacements of sliders 1 and 2 are obtained by solving the intersections of two circles with a center $P_{1}$ and radii $P_{1} B_{1}$ and $P_{2} B_{2}$ and a line $Z=0$, respectively. When the orientation of the output link is given in addition, the input displacement of slider 3 is obtained by solving the intersection of a circle with a center $\mathrm{P}_{3}$ and radius $P_{3} B_{3}$ and a line $Z=0$. And, the workspace with respect to $P_{1}$ is determined only by the part $B_{1} P_{1}\left(P_{2}\right) B_{2}$, which is also shown in the figure taking into account the motion range constraints of prismatic joints 1 and 2. As for the statics, the external load applied to the output link is supported by the tension/compression forces of links $\mathrm{B}_{1} \mathrm{P}_{1}, \mathrm{~B}_{2} \mathrm{P}_{2}$ and $\mathrm{B}_{3} \mathrm{P}_{3}$. To simplify, some criteria for kinematic design are set. Two links $\left(\mathrm{B}_{1} \mathrm{P}_{1}\right.$ and $\left.\mathrm{B}_{2} \mathrm{P}_{2}\right)$ are equal and share the same rotation axis, and prismatic joints 1 and 2 are placed to make the workspace of $\mathrm{P}_{1}$, the blue area in Fig. 7. Transmission angle $\phi_{1}$ between Links $\mathrm{B}_{1} \mathrm{P}_{1}$ and $\mathrm{B}_{2} \mathrm{P}_{2}$ is limited to the range $\left[60^{\circ} 120^{\circ}\right]$, and transmission angle $\phi_{3}$ between link $\mathrm{B}_{3} \mathrm{P}_{3}$ and the footplate is limited to the range $\left[30^{\circ} 150^{\circ}\right.$ ] for ensuring the efficient force transmission. The requirements and criteria for the design of the parallel mechanism are summarized as follows:

( 1 ) Desired $\mathrm{P}_{1}$ trajectories should be covered by the workspace by the part of $\mathrm{B}_{1} \mathrm{P}_{1}\left(\mathrm{P}_{2}\right) \mathrm{B}_{2}$.

( 2 ) Desired $\mathrm{P}_{3}$ trajectories should be covered by the part of $\mathrm{P}_{1} \mathrm{P}_{3} \mathrm{~B}_{3}$.

( 3 ) Transmission angles for the all trajectories should satisfy the conditions $\phi_{1}:\left[60^{\circ}, 120^{\circ}\right], \phi_{3}:\left[30^{\circ}, 150^{\circ}\right]$.

Table 1 The height and weight of subjects tested

\begin{tabular}{ccccc} 
Subjects & 1 & 2 & 3 & 4 \\
\hline Height $(\mathrm{cm})$ & 167 & 159 & 176 & 182 \\
Mass $(\mathrm{kg})$ & 56.0 & 50.4 & 77.0 & 72.5
\end{tabular}

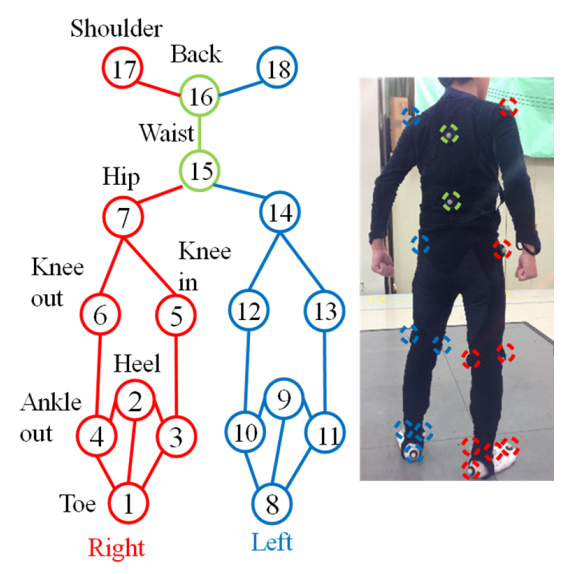

Fig. 4 Positions of the markers during the motion capture experiments

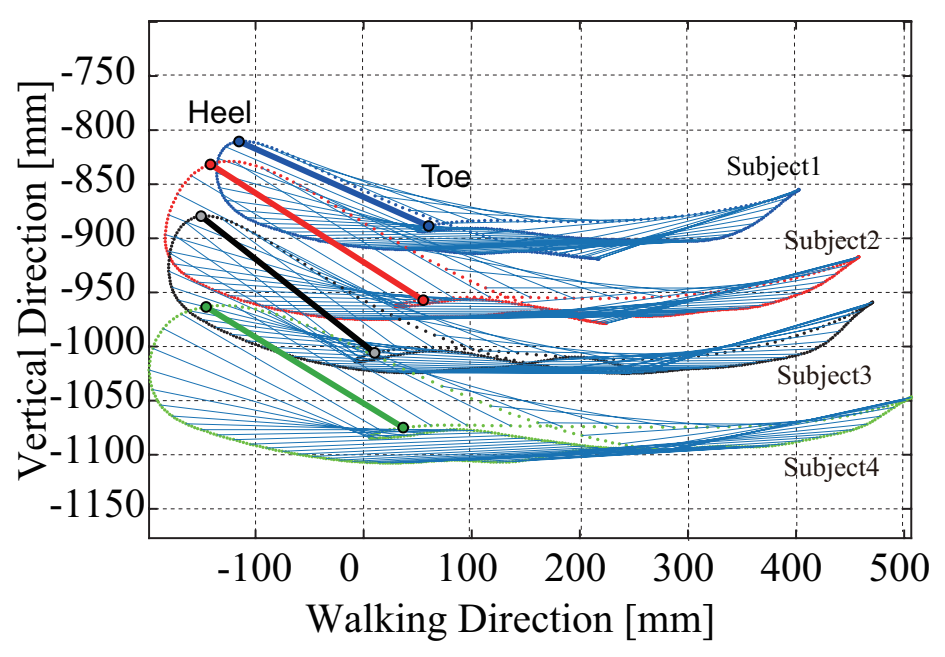

Fig. 5 Trajectories of the foot (heel and toe) for the 4 subjects with respect to the body coordinate system 


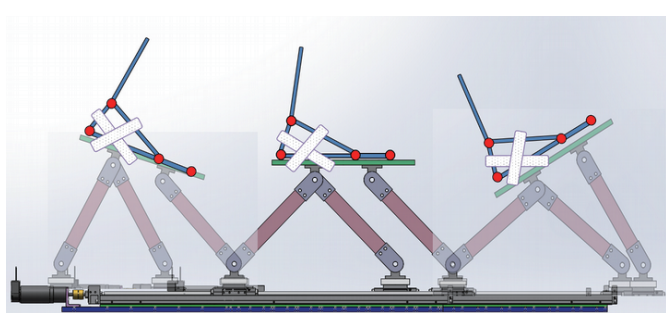

(a) Method 1 attaching hind foot onto the foot-plate

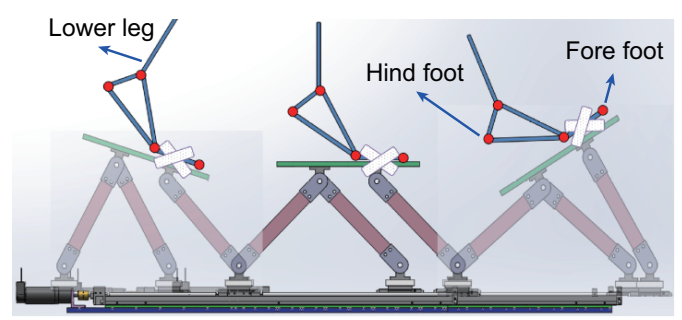

(b) Method 2 only attaching fore foot onto the foot-plate

Fig. 6 Two different design method for attachment of foot onto the foot-plate

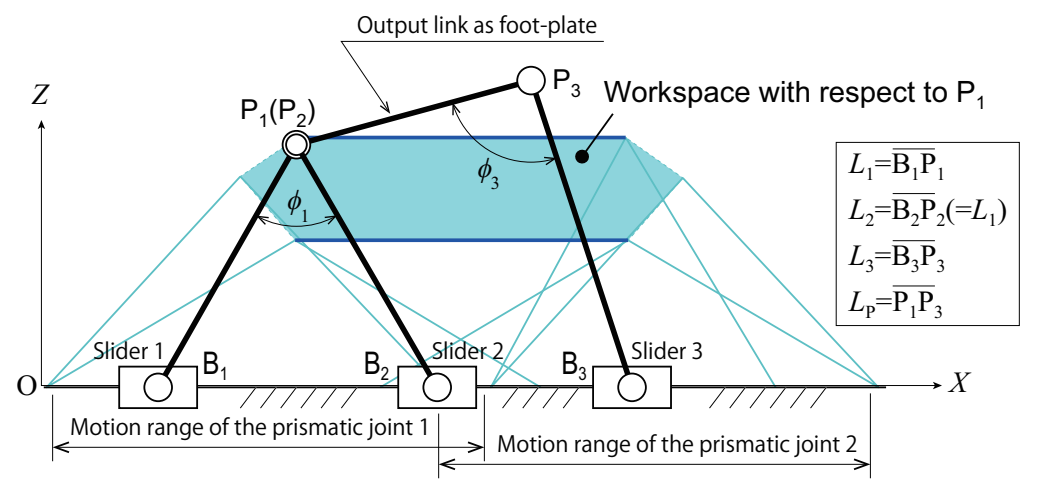

Fig. 7 Kinematic diagram of planar 3-PRR mechanism and its workspace under constraint on the motion range of prismatic joints

The load force applied to the foot-plate is mainly supported by the partial mechanism $\mathrm{B}_{1} \mathrm{P}_{1}\left(\mathrm{P}_{2}\right) \mathrm{B}_{2}$ and the part $\mathrm{B}_{3} \mathrm{P}_{3} \mathrm{P}_{1}\left(\mathrm{P}_{2}\right)$ plays a role to change the orientation supporting the moment around $\mathrm{P}_{1}$. Since the reference of the moment support is considered to be $\mathrm{P}_{1}$, the position of $\mathrm{P}_{1}$ should be rigidly kept against external load force. Then, the condition of the transmission angle $\phi_{1}$ is set severer.

Based on the needs and design criteria above, required mechanical components specifications and dimensions can be obtained. In the optimization of the kinematic parameters, we changed the parameters value repeatedly to find the optimal one. After finding the length of footplate and links meeting the requirements, its quality for achieving the required gait motion was evaluated based on transmission angle. The flowchart of the optimization process is shown in Fig. 8 .

Firstly, we get the desired trajectory of $\mathrm{P}_{1}$ from the gait data and footplate length, adjust the trajectories into workspace of $\mathrm{P}_{1}$ by considering criteria above. Since $\mathrm{P}_{3}$ will move with $\mathrm{P}_{1}$ trajectories, a possible link length $L_{3}$ based on their coordinates along Z-axis can be achieved. Finally, the transmission angles can be used to check whether they fit requirements.

As all curves should be perfectly covered by the joint's workspace as shown in Fig. 9a, the position of $\mathrm{P}_{1}$ and the new $\mathrm{P}_{3}$ trajectories needed to be modified. The desired traces of $\mathrm{P}_{3}$ on the footplate are generated as Fig. $9 \mathrm{~b}$. As a result, the necessary link length $L_{1}\left(=L_{2}\right)$ is $410 \mathrm{~mm}$, which makes workspace of $\mathrm{P}_{1}$ larger than $150 \mathrm{~mm}$ in vertical direction and all the desired $\mathrm{P}_{1}$ trajectories are reachable.

Not only the desired $\mathrm{P}_{1}$ trajectories should be covered by its workspace, trajectories of $\mathrm{P}_{3}$ also need to be checked. The link length $L_{3}$ should be longer than the vertical direction displacement of all 4 cases and make the transmission angle smaller than $150^{\circ}$ as well. After adjusting the length of footplate and links meeting the requirements, the longest lengths of $L_{p}$ (footplate) and $L_{3}$ was found. After these analysis, the links' length were optimized as $410 \mathrm{~mm}$ and $303 \mathrm{~mm}$, and the distance between the joints on the footplate is $100 \mathrm{~mm}$. Furthermore, the limited transmission angles change as the footplate moves, which are shown in Fig. 10a and 10b. With these parameters, the desired trajectories could be generated by the machine and reach the design assumptions.

The second method, however, needs to consider more details on the foot. As there are metatarsal bones between heel and toe joints, during the heel off stage of a typical gait cycle, metatarsal and toe joint support and accelerate the leg together. If the device supports and actuates patients' feet in such way, it is much closer to the natural way of walking. According to the data of average length of the foot anatomy in Japan provided by AIST, forefoot occupies around 33\% of 


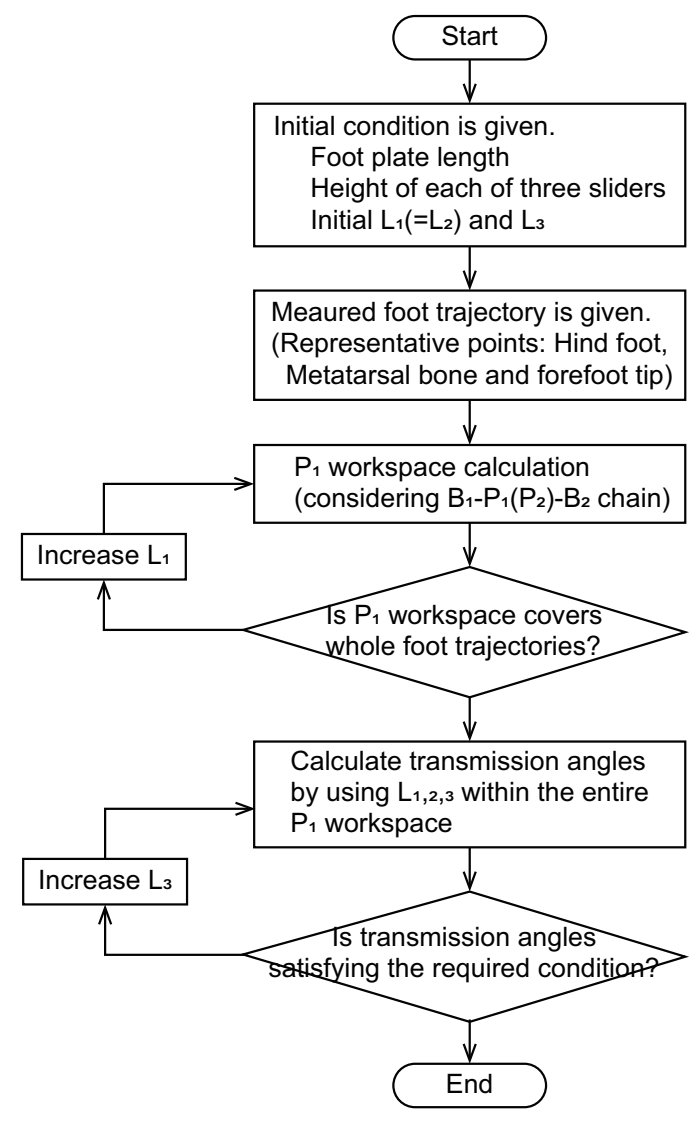

Fig. 8 Flowchart of the optimization process of the essential kinematic parameters

the whole foot (AIST, accessed on 1 January, 2016).

From trajectories of heel and toe, Fig. 11 illustrates how the foot moves in one gait cycle. In the figure, the subject is walking toward right and the coordinates' origin is at its center of mass. On the left, there are heel off and metatarsal joint off the ground obviously. During the period of heel off, the foot pushes off the ground and accelerates, while the center of mass moves upward and forward, which explains why the curve goes down rapidly in this phase. Same as the algorithm used in the last method, trajectories of metatarsal joint and toe are supposed to be generated with the designed footplate. In Fig. 9c and 9d, since all the four desired $\mathrm{P}_{1}$ trajectories were adjusted into the workspace of it, the $\mathrm{P}_{2}$ trajectories were also obtained. As a result, $L_{1}$ and $L_{3}$ were optimized to be $240 \mathrm{~mm}$, and the footplate is $120 \mathrm{~mm}$. If considering the height difference of the linear guides, we can put the Guide2 10, 20, or $30 \mathrm{~mm}$ up or down, and then compare the influence on the required workspace size and input velocity of motor 2 to drive the prismatic joint 2 . However, it makes almost no difference compared with the original height. Thus, we made the three linear guides be put on the same height.

Comparing the above methods (Fig. 9a and 9c) through Fig. 12, the latter method results in more compact design. Due to the shorter links, the machine will be more stable, more precise, safer, lighter and costs less. Users can choose a healthy individual's gait pattern as reference based on the body conditions of their own. The machine can offer both large and small stride modes. Also, it is possible for them to choose different walking velocities as they prefer.

\section{Prototype}

After the above-mentioned analysis works, a prototype, as shown in Fig. 13, was built for testing. Specifications of important components and representative link length of the mechanism are shown in Table 2. The prototype consists of three sections. First, the mechanism section, the main mechanical components are three linear sliders, three links and a platform, which are linked by some revolute joints. Furthermore, the machine is driven by three DC motors controlled by a computer as shown in Fig. 14. This is the electronic section. Lastly, the control program section. The control box is linked to the PC and receives command signals, and there is a control program transforming the walking data into motor 
Zhang, Lan, Matsuura, Mougenot, Sugahara and Takeda,

Journal of Advanced Mechanical Design, Systems, and Manufacturing, Vol.12, No.1 (2018)

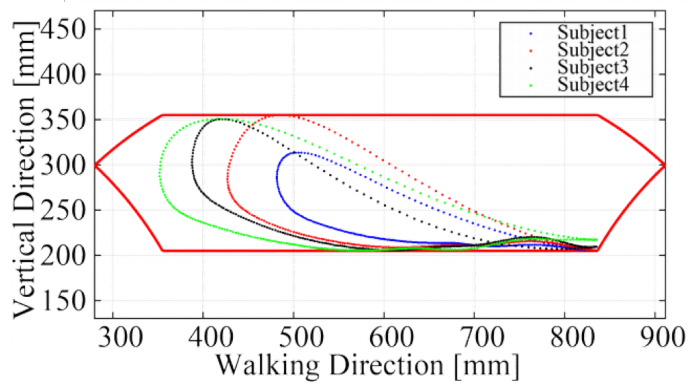

(a) Method 1: workspace and $\mathrm{P}_{1}$ trajectories

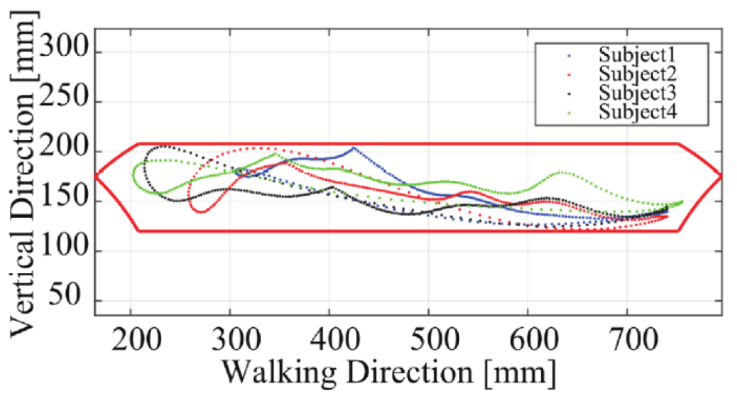

(c) Method 2: workspace and $\mathrm{P}_{1}$ trajectories

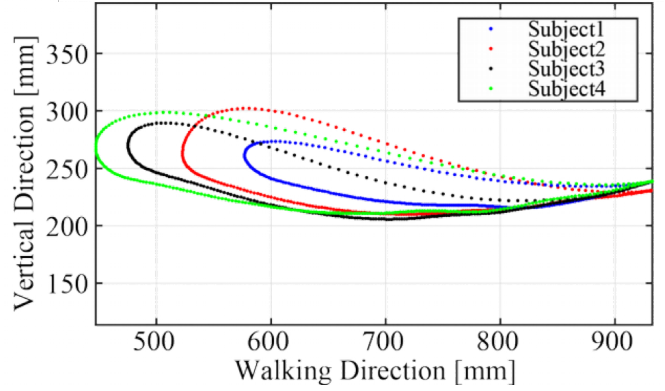

(b) Method 1: desired $\mathrm{P}_{1}$ and $\mathrm{P}_{3}$ trajectories

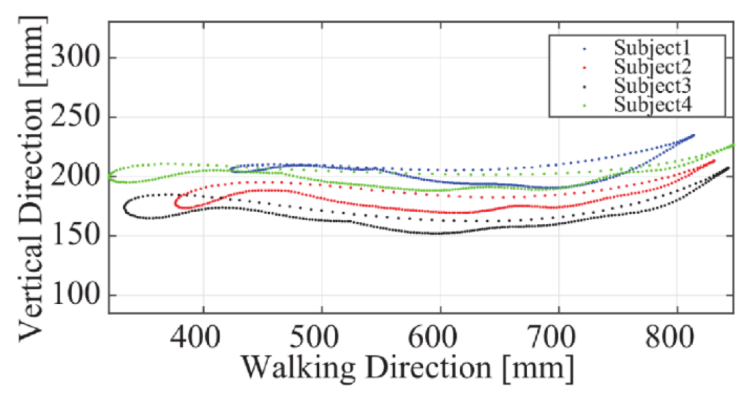

(d) Method 2: desired $\mathrm{P}_{1}$ and $\mathrm{P}_{3}$ trajectories

Fig. 9 Desired trajectories and workspace of $\mathrm{P}_{1}$, and trajectories of $\mathrm{P}_{3}$

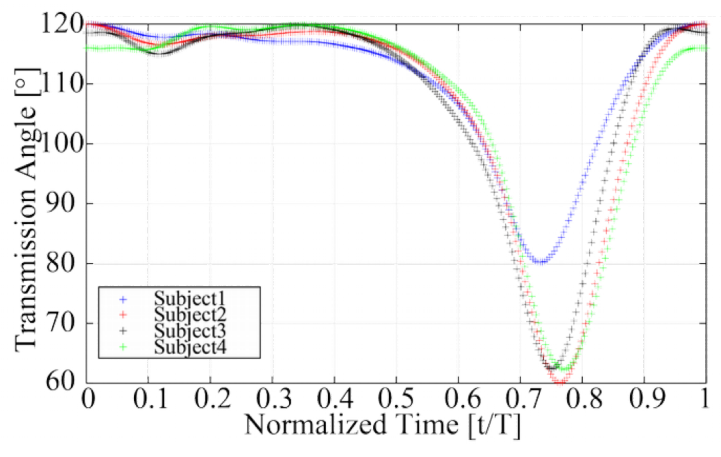

(a) Method 1: transmission angle $\phi_{1}$

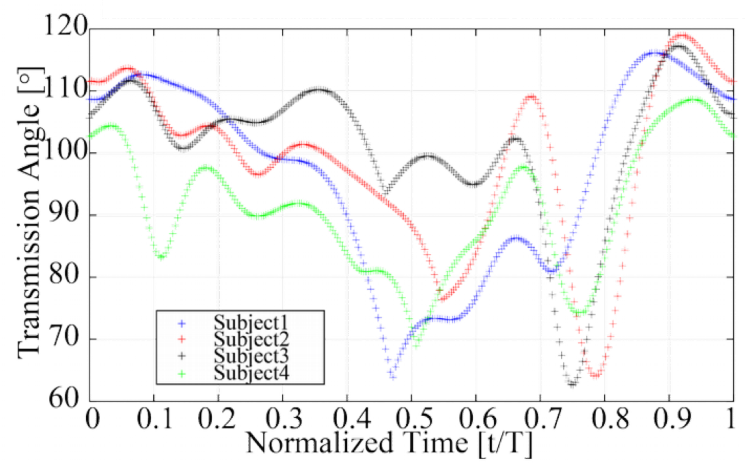

(c) Method 2: transmission angle $\phi_{1}$

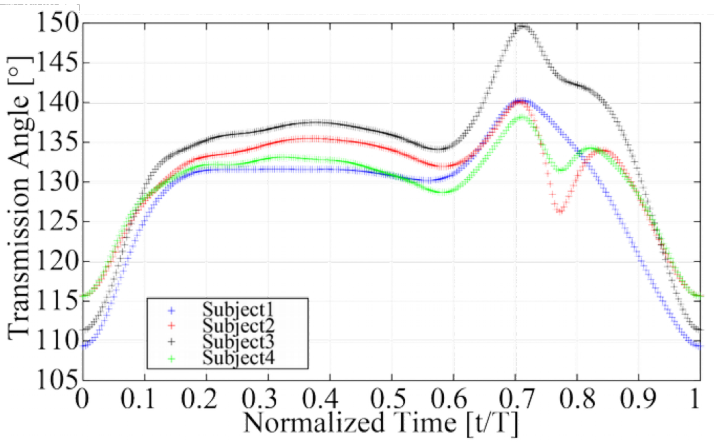

(b) Method 1: transmission angle $\phi_{3}$

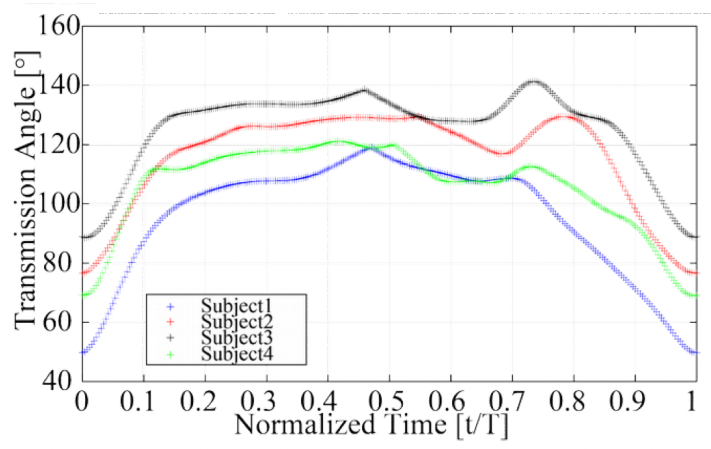

(d) Method 2: transmission angle $\phi_{3}$

Fig. 10 Transmission angles 


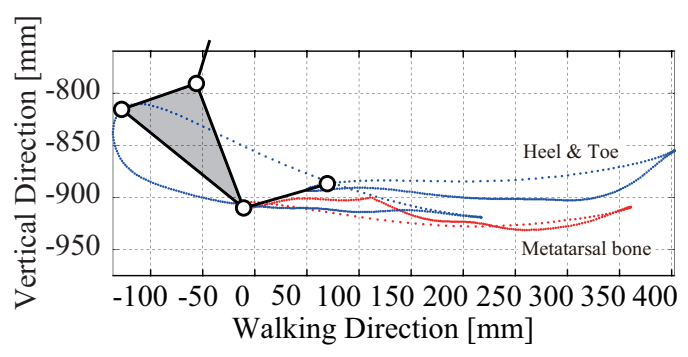

Fig. 11 Trajectories of the heel, metatarsal, toe joints

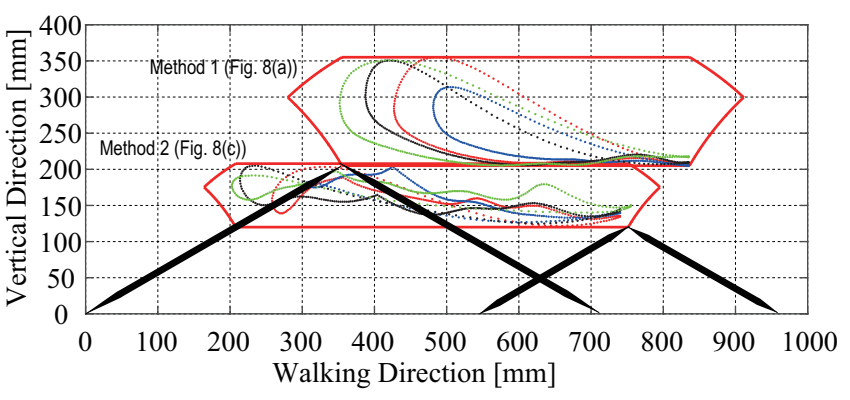

Fig. 12 Comparison of the workspace for two design methods

control data with inverse kinematics. From the PC program, control signals are converted through the boxes and send commands to the motors, then the footplate will generate the desired walking patterns we want.

Table 2 Major specifications of the prototype

\begin{tabular}{|c|l|}
\hline & $\begin{array}{l}\text { DC servo motor of 24V/90W (RE35 Maxon Motor ag.) } \\
\text { Planetary gear head of 4.8:1 reduction ratio (GP32A, Maxon Motor ag.) } \\
\text { Optical encoder with 500PPR resolution (HEDL5540, Maxon Motor ag.) } \\
\text { Binear actuator }\end{array}$ \\
\hline Bink length screw of 730mm long with 20mm lead with a pair of sliders (Monocarrier MCM05060H20K00, NSK Ltd.) \\
\hline$L_{1}, L_{2}, L_{3}=240 \mathrm{~mm}, L_{P}=120 \mathrm{~mm}$ \\
\hline
\end{tabular}

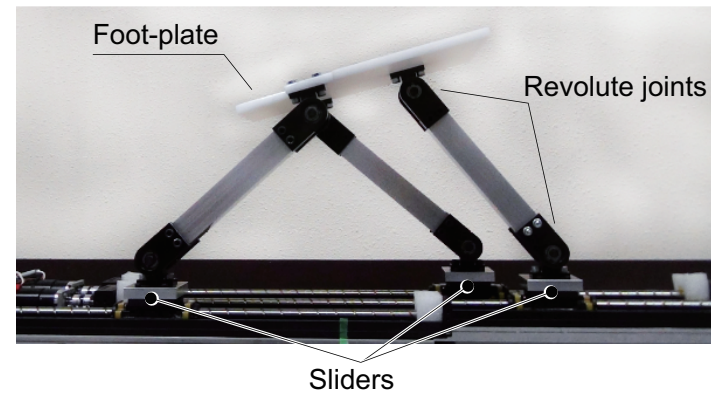

Fig. 13 Prototype of the machine

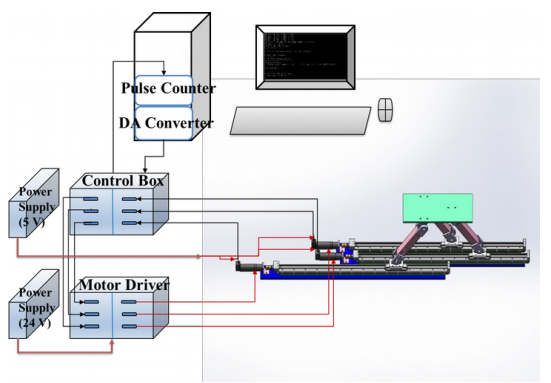

Fig. 14 The PC controlled system

In the mechanism design, the configuration obtained by the above analysis scheme with the Method 2 was employed to detemine the necessary link length. Components of the linear actuator was chosen to achieve necessary velocity and torque of the prismatic joint. In the motion control of the machine, reference position trajectory of each actuator was obtained by solving the inverse kinematics. For the obtained reference trajectory of each actuator, PI feedback control was applied. The output position of each slider was acquired by an optical encoder. During several experiments, the parameters of the controller were tuned to minimize the error between input and output. In Fig. 15, the $D$ and $N$ stand for disturbance and noise respectively. $K_{\mathrm{p}}$ and $K_{\mathrm{i}}$, denoted the coefficients for proportional and integral terms, which would provide a smooth working.

After collecting the position data from the user test, in Fig. 16, the result is putting together with the original dataset and can be found that it perfectly matches the initial data. Let us note that we observed a contact loss of the heel with the foot-plate at around the heel-off phase during the experiments, as that was expected in the analysis process. This result demonstrates the effectiveness of the proposed design.

\section{Conclusions}

In the present study, design approach of planar 3-PRR parallel mechanism for a foot-plate drive mechanism with a compact size for gait rehabilitation was proposed. To this end, an idea of the placement and fixation of the foot on the 


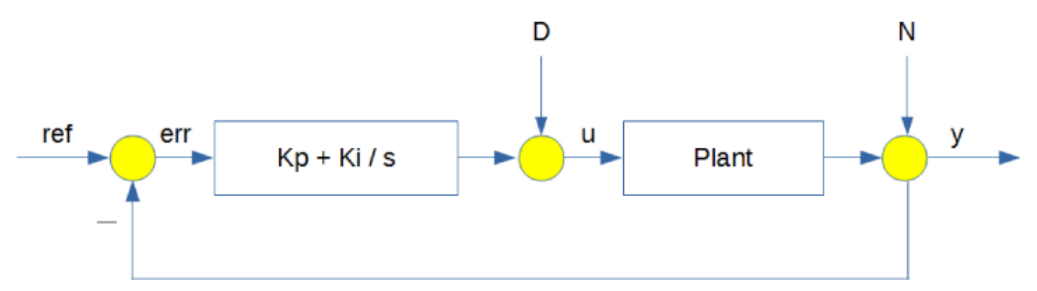

Fig. 15 Block diagram of control system

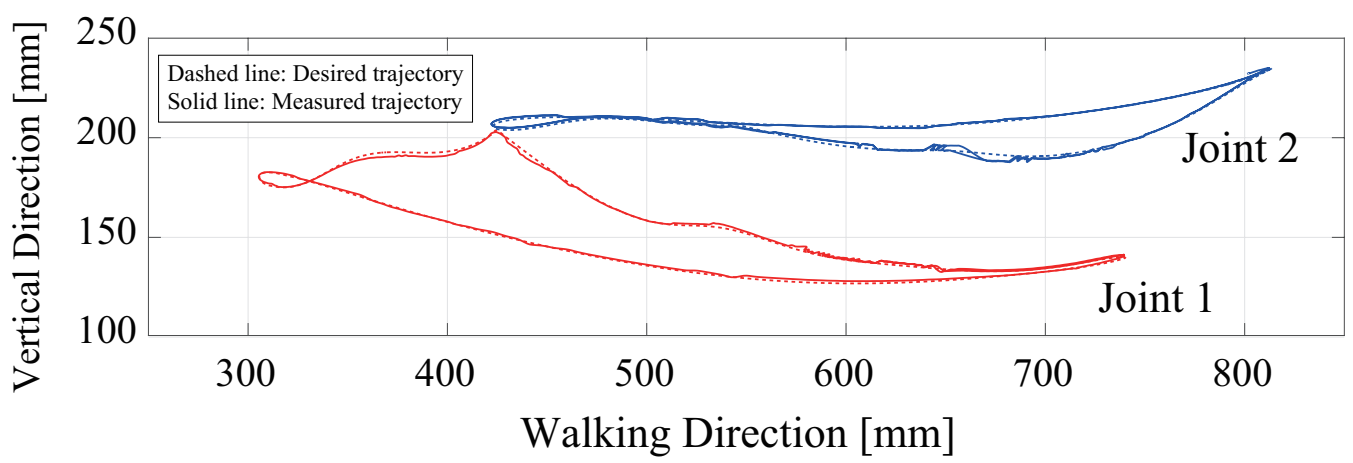

Fig. 16 Comparison of reference and measured trajectories obtained in experiment using the prototype with method 2

foot-plate was proposed, and design procedure was concretely shown. The patients are supposed to be supported mainly by a harness system with their feet fastened onto two separated footplates that can be programmed to generate gait patterns with different sizes and velocities. Based on processed walking experiments data on healthy subjects, a 3-PRR parallel mechanism was proposed to drive the footplate.

The mechanical specifications were determined during the gait analysis, with the reachable workspace and motion transmissibility taken into consideration. The method of attaching the forefoot on the plate significantly optimized the design of the mechanism, having made the designed mechanism smaller.

While this research provides the basic framework for making a gait rehabilitation system, more and more walkingdisabled people could train their walking ability with the help of this device. However, more work is needed in several areas to improve the designed machine. For example, more communication between the researchers and the target users should be made, a complete version of the designed rehabilitation system should be built, and more tests on patients should be done if safety could be guaranteed.

\section{Acknowledgement}

We would like to thank Dr. Hiroshi Ujiie and Dr. Tomokazu Takakura with Tokyo Rosai Hospital for giving us advises from medical and clinical points of view and Mr. Tomohiro Suzuki and Mr. Yuta Chounan for assisting with motion capture experiments that greatly improved the research.

\section{References}

AIST, Japanese Body Dimension Data (1997-98), Dh.aist.go.jp (online), available from <https://www.dh.aist.go.jp/ database/97-98/e_index.html>, (accessed on 1 Junuary, 2016).

Bonev, I., Zlatanov, D. and Gosselin, C., Singularity Analysis of 3-DOF Planar Parallel Mechanisms via Screw Theory, Journal of Mechanical Design, Vol.125, No.3 (2003), pp.573-581.

Colombo, G., Joerg, M., Schreier, R. and Dietz, V., Treadmill training of paraplegic patients using a robotic orthosis, Journal of Rehabilitation Research and Development, Vol.37 (2000), pp.693-700.

Harada, T. and Nagase, M., Configurations and Mathematical Models of Parallel Link Mechanisms Using Multi Drive 
Linear Motors, Proceedings of the 2009 IEEE/RSJ international conference on Intelligent robots and systems, (2009), pp.1974-1979.

Hesse, S. and Uhlenbrock, D., A mechanized gait trainer for restoration of gait, Journal of Rehabilitation Research and Development, Vol.37 (2000), pp.701-708.

Kawamoto, H. and Sankai, Y., Power Assist System HAL-3 for Gait Disorder Person, Proceedings of International Conference on Computers for Handicapped Persons, (2002), pp.196-203.

Matsuura, D., Funato, R., Chounan, Y., Tharasrisuthi, K. and Takeda, Y., Synthesis and Analysis of the Mechanism for Walking Assist Machine for Persons with Hemiplegia, The 14th Mechatronics Forum International Conference (Mechatronics 2014), (2014), pp. 555-562.

Matsuura, D., Funato, R., Ogata, M., Higuchi, M. and Takeda, Y., Efficiency Improvement of Walking Assist Machine using Crutches Based on Gait-Feasible Region Analysis, Mechanism and Machine Theory, Vol.84 (2015), pp.126133.

Precor Inc., AMT 885 - The All-New Adaptive Motion Trainer (AMT) from Precor, Precor.com (online), available from $<$ https://www.precor.com/en-us/commercial/cardio/amt\#amt-open-stride>, (accessed on 1 Junuary 2016).

Schmidt, H., Hesse, S., Bernhardt, R. and Krüger, J., HapticWalker-a novel haptic foot device, ACM Transactions on Applied Perception, Vol.2, No.2 (2005), pp.166-180.

Wang, J. and Gosselin, C. M., Kinematic Analysis and Design of Kinematically Redundant Parallel Mechanisms, Journal of Mechanical Design, Vol.126, No.1 (2004), pp.109-118.

Yoon, J. W., Novandy, B., Yoon, C. H. and Park, K. J., A 6-DOF Gait Rehabilitation Robot With Upper and Lower Limb Connections That Allows Walking Velocity Updates on Various Terrains, IEEE/ASME Transactions on Mechatronics, Vol.15 (2010), pp.201-215. 